\title{
Upper Bounds on the BER Performance of MTCM-STBC Schemes over Shadowed Rician Fading Channels
}

\author{
M. Uysal \\ Department of Electrical and Computer Engineering, University of Waterloo, Waterloo, ON, Canada N2L 3G1 \\ Email:muysal@ece.uwaterloo.ca \\ C. N. Georghiades \\ Department of Electrical Engineering, Texas A\&M University, College Station, TX 77843-3128, USA \\ Email: georghiades@ee.tamu.edu
}

Received 17 May 2003; Revised 21 October 2003

\begin{abstract}
Space-time block coding (STBC) provides substantial diversity advantages with a low decoding complexity. However, these codes are not designed to achieve coding gains. Outer codes should be concatenated with STBC to provide additional coding gain. In this paper, we analyze the performance of concatenated trellis-coded STBC schemes over shadowed Rician frequency-flat fading channels. We derive an exact pairwise error probability (PEP) expression that reveals the dominant factors affecting performance. Based on the derived PEP, in conjunction with the transfer function technique, we also present upper bounds on the bit error rate (BER), which are further shown to be tight through a Monte-Carlo simulation study.
\end{abstract}

Keywords and phrases: space-time block coding, trellis-coded modulation, Rician fading channels, shadowing, pairwise error probability.

\section{INTRODUCTION}

Space-time trellis coding was introduced in [1] as an effective transmit diversity technique to combat fading. These codes were designed to achieve maximum diversity gain. However, for a fixed number of transmit antennas, their decoding complexity increases exponentially with the transmission rate. Space-time block coding (STBC) [2] was proposed as an attractive alternative to its trellis counterpart with a much lower decoding complexity. The work in [2] was inspired by Alamouti's early work [3], where a simple two-branch transmit diversity scheme was presented and shown to provide the same diversity order as maximal-ratio receiver combining with two receive antennas. Alamouti's scheme is appealing in terms of its performance and simplicity. Assuming the channel is known at the receiver, it requires a simple maximumlikelihood decoding algorithm based only on linear processing at the receiver. STBC generalizes Alamouti's scheme to an arbitrary number of transmit antennas and is able to provide the full diversity promised by the transmit and receive antennas. However, these codes are not designed to achieve a coding gain. Therefore, outer codes should be concatenated with STBC to achieve additional coding gains. A pioneering work towards this end is presented in [4] where concatenation of trellis-coded modulation (TCM) with STBC is considered. In [4], it is shown that the free distance of the trellis code dominates performance; therefore, the optimal trellis codes designed for additive white Gaussian noise (AWGN) are also optimum for concatenated TCM-STBC over quasistatic Rayleigh fading channels. We studied the same concatenated scheme combined with an interleaver in [5] over Rician fading channels. In this paper, we generalize our work to shadowed Rician channels. The shadowed Rician channel [6] is a generalization of the Rician model, where the line-of-sight (LOS) path is subjected to a lognormal transformation due to foliage attenuation or blockage, also referred to as shadowing. Specifically, we derive an exact pairwise error probability (PEP) for concatenated TCM-STBC schemes. Our exact evaluation of PEP is based on the moment-generating function technique $[7,8]$, which has been successfully applied to the analysis of digital communication systems over fading channels. Using the classical transfer function technique based on the exact PEP, we obtain upper bounds on bit error rate (BER) performance, which are further verified through simulation. Our analysis also reveals the selection criteria for trellis codes which should be used in conjunction with STBC.

The organization of the paper is as follows. In Section 2 we explain our system model, where the concatenated TCMSTBC is described and the channel model under consideration is introduced. In Section 3 an exact expression for PEP is derived for the TCM-STBC scheme using the MGF approach. Based on the derived PEP, we discuss the selection criteria 
for trellis codes which should be used with space-time codes for optimal performance and compare them with the classical selection criteria for trellis codes over fading channels without transmitter diversity. In Section 4, using the transfer function technique in conjunction with the derived PEP expressions, we obtain upper bounds on the BER performance. Analytical performance results are presented for two example trellis codes, which are further confirmed through MonteCarlo simulation.

\section{SYSTEM MODEL}

We consider a wireless communication scenario where the transmitter is equipped with $M$ antennas and the receiver is equipped with $N$ antennas. The binary data is first encoded by a trellis encoder. After trellis coded symbols are interleaved and mapped to constellation symbols, they are fed to the STBC encoder. An STBC is defined [2] by an $L \times M$ code matrix, where $L$ represents the number of time intervals for transmitting $P$ symbols, resulting in a code rate of $P / L$. For Tarokh et al.'s orthogonal space-time block codes [2], the entries of the code matrix are chosen as linear combinations of the transmission symbols and their conjugates. For example, the code matrix for the well-known Alamouti's scheme (i.e., STBC for 2 transmit antennas) is given by

$$
\left[\begin{array}{cc}
x_{1} & x_{2} \\
-x_{2}^{*} & x_{1}^{*}
\end{array}\right]
$$

with $M=P=L=2$.

We assume that the transmission frame from each antenna consists of a total of $F L$ symbols (i.e., consecutive $F$ smaller inner-frames, each of them having duration $L$ symbols corresponding to the STBC length). The received signal at receive antenna $n(n=1,2, \ldots, N)$ at time interval $l$ of the $f$ th $(f=1,2, \ldots, F)$ inner-frame is a superposition of $M$ transmitted signals:

$$
r_{n}^{f}(l)=\sum_{m=1}^{M} \alpha_{m, n}^{f} x_{m}^{f}(l)+\eta_{n}^{f}(l),
$$

where $x_{m}^{f}(l)$ is the modulation symbol transmitted from the $m$ th transmit antenna at time interval $l$ of the $f$ th frame and $\eta_{n}^{f}(l)$ is additive noise, modeled as a complex Gaussian random variable with zero mean and variance $N_{0} / 2$ per dimension. $\alpha_{m, n}^{f}$ represents the fading coefficient modeling the channel from the $m$ th transmit to the $n$th receive antenna during the $f$ th inner frame and are assumed to be independent and identically distributed (i.i.d.). The fading coefficient is assumed to remain constant over an inner-frame period (i.e., $L$ symbol intervals). This assumption is necessary to make use of the orthogonal structure of STBC to guarantee full spatial diversity. The assumption of quasistatic behavior of the channel over an inner-frame period can be justified using an $L$-symbol interleaver over a moderately slow varying channel. In our case, the fading amplitude is described by the shadowed Rician fading model. In this model, the
LOS component is not constant but rather a lognormally distributed random variable. The fading coefficient can be expressed (dropping the subscripts and superscripts for notational convenience) as $\alpha=\mu+\xi_{0}+j \xi_{1}$, where $\xi_{0}$ and $\xi_{1}$ are independent Gaussian random variables with zero mean and variance $\sigma^{2}$. Here, the LOS component is given as $\mu=\exp \left(\xi_{2}\right)$ where $\xi_{2}$ is a Gaussian random variable with mean $m_{\mu}$ and variance $\sigma_{\mu}^{2}$, and independent of $\xi_{0}$ and $\xi_{1}$. The conditional probability density function of the fading amplitude $|\alpha|$ is

$$
\begin{aligned}
& p_{|\alpha| \mid \mu}(|\alpha| \mid \mu) \\
& =\frac{|\alpha|}{\sigma^{2}} \exp \left(-\frac{|\alpha|^{2}+\mu^{2}}{2 \sigma^{2}}\right) I_{0}\left(\frac{|\alpha| \mu}{\sigma^{2}}\right), \quad|\alpha| \geq 0,
\end{aligned}
$$

where $I_{0}(\cdot)$ is the zero-order modified Bessel function of the first kind, and the probability density function of the LOS component is given by

$$
p_{\mu}(\mu)=\frac{1}{\sqrt{2 \pi} \sigma_{\mu} \mu} \exp \left(-\frac{\left(\ln \mu-m_{\mu}\right)^{2}}{2 \sigma_{\mu}^{2}}\right) .
$$

The parameters $\sigma, \sigma_{\mu}$, and $m_{\mu}$ in (3) and (4) specify the degree of shadowing. Denoting by $\mathbb{C}^{m \times n}$ the vector space of $m$ by- $n$ complex matrices, and defining ${ }^{1}$

$$
\begin{aligned}
\mathbf{r}_{n}^{f} & =\left(r_{n}^{f}(1), r_{n}^{f}(2), \ldots, r_{n}^{f}(L)\right)^{T} \in \mathbb{C}^{L \times 1}, \\
\boldsymbol{\alpha}_{n}^{f} & =\left(\alpha_{1, n}^{f}, \alpha_{2, n}^{f}, \ldots, \alpha_{M, n}^{f}\right)^{T} \in \mathbb{C}^{M \times 1}, \\
\boldsymbol{\eta}_{n}^{f} & =\left(\eta_{n}^{f}(1), \eta_{n}^{f}(2), \ldots, \eta_{n}^{f}(L)\right)^{T} \in \mathbb{C}^{L \times 1},
\end{aligned}
$$

the received signal can be written in matrix notation as

$$
\mathbf{r}_{n}^{f}=\mathbf{X}^{f} \boldsymbol{\alpha}_{n}^{f}+\boldsymbol{\eta}_{n}^{f}, \quad n=1,2, \ldots, N, f=1,2, \ldots, F,
$$

where $\mathbf{X}^{f} \in \mathbb{C}^{L \times M}$ consists of space-time encoded symbols (which have been already trellis encoded) for the $f$ th inner frame. At the receiver, first the received signal is passed through the space-time decoder, which is essentially based on linear processing for STBC from orthogonal designs [2]. After deinterleaving, the processed sequence is fed to the trellis decoder implemented by a Viterbi algorithm. If a multiple TCM (MTCM) scheme with $M$ symbols per branch is used (note that the number of transmit antennas is also given as $M)$, the decoding steps can be combined in one step with a proper modification of the metric employed in the Viterbi algorithm. In this case, the received signal is just deinterleaved and fed directly to the Viterbi decoder without any further processing.

\section{DERIVATION OF EXACT PEP}

In this section, we analyze the PEP of the concatenated scheme over shadowed Rician fading channels assuming

\footnotetext{
${ }^{1}$ Throughout this paper, we use $(\cdot)^{T}$ and $(\cdot)^{H}$ for the transpose and transpose conjugate operations, respectively. Upper case bold face letters represent matrices and lower case bold face letters represent vectors.
} 
perfect channel state information is available at the receiver. Assuming equal transmitted power at all transmit antennas, the conditional PEP of transmitting code matrix $\mathbf{X}$ (which consists of $\mathbf{X}^{f}, f=1,2, \ldots, F$ ) and erroneously deciding in favor of another code matrix $\hat{\mathbf{X}}$ at the decoder is given by

$$
\begin{array}{r}
P\left(\mathbf{X}, \hat{\mathbf{X}} \mid \alpha_{m, n}^{f}, \mu_{m, n}^{f}, m=1, \ldots, M,\right. \\
n=1, \ldots, N, f=1, \ldots, F) \\
=Q\left(\sqrt{\sum_{f=1}^{F} \sum_{n=1}^{N}\left(\boldsymbol{\alpha}_{n}^{f}\right)^{H} \mathbf{A}^{f} \boldsymbol{\alpha}_{n}^{f}}\right),
\end{array}
$$

where $Q(\cdot)$ is the Gaussian $Q$-function and $\mathbf{A}^{f}$ is given by

$$
\mathbf{A}^{f}=\frac{1}{M} \frac{E_{s}}{2 N_{0}}\left(\mathbf{X}^{f}-\hat{\mathbf{X}}^{f}\right)^{H}\left(\mathbf{X}^{f}-\hat{\mathbf{X}}^{f}\right)
$$

Here, $E_{s}$ is the total signal power transmitted from all $M$ transmit antennas and $N_{0} / 2$ is the noise variance per dimension. In order to find the unconditional PEP, we need to take expectations with respect to $\alpha_{m, n}^{f}$ and $\mu_{m, n}^{f}$. The expectation with respect to fading coefficients can be obtained through use of the alternative form of the Gaussian Q-function [8] as

$$
\begin{aligned}
& P\left(\mathbf{X}, \hat{\mathbf{X}} \mid \mu_{m, n}^{f}, m=1, \ldots, M, n=1, \ldots, N, f=1, \ldots, F\right) \\
& \quad=\frac{1}{\pi} \int_{0}^{\pi / 2} \Phi_{\Gamma}\left(-\frac{1}{2 \sin ^{2} \theta}\right) d \theta,
\end{aligned}
$$

where $\Phi_{\Gamma}(s)$ is the moment generating function (MGF) of

$$
\Gamma=\sum_{f=1}^{F} \sum_{n=1}^{N}\left(\boldsymbol{\alpha}_{n}^{f}\right)^{H} \mathbf{A}^{f} \boldsymbol{\alpha}_{n}^{f}
$$

$\Gamma$ is a quadratic form of complex Gaussian random variables and its MGF is given as $[9,10]$

$$
\Phi_{\Gamma}(s)=\prod_{f=1}^{F} \prod_{n=1}^{N} \prod_{m=1}^{M} \frac{1}{1-s \chi_{m}} \exp \left(\frac{s \chi_{m}\left|d_{m}\right|^{2}}{1-s \chi_{m}}\right),
$$

where $\chi_{m}$ are the eigenvalues of $\boldsymbol{\Sigma} \mathbf{A}^{f}$ and $d_{m}$ are the elements of $M$-length vector $\mathbf{d}=\boldsymbol{\mu} \boldsymbol{\Sigma}^{-1 / 2}$. Here $\boldsymbol{\mu}$ and $\boldsymbol{\Sigma}$ represent the mean vector and the covariance matrix of $\boldsymbol{\alpha}_{n}^{f}$, respectively. Making use of the assumed i.i.d. properties of the fading channel, we obtain $\left|d_{m}\right|^{2}=\mu^{2} / 2 \sigma^{2}$. Furthermore, in our case, $\mathbf{A}^{f}$ is a diagonal matrix due to the orthogonality of STBC and the eigenvalues $\chi_{m}$ are simply equal to the diagonal elements of $\Sigma \mathbf{A}^{f}$, that is,

$$
\frac{E_{s}}{2 N_{0}} 2 \sigma^{2} \frac{\beta}{M} \sum_{p=1}^{P}\left|x_{p}^{f}-\hat{x}_{p}^{f}\right|^{2}
$$

where $\beta=1$ for $M=2$ and $\beta=2$ for $M>2$ due to the special matrix structure of STBC based on orthogonal designs [2]. Inserting (11) into (9) and using the i.i.d. properties for fading coefficients, we obtain

$$
\begin{aligned}
& P(\mathbf{X}, \hat{\mathbf{X}} \mid \mu) \\
& =\frac{1}{\pi} \int_{0}^{\pi / 2} \prod_{f=1}^{F}\left[\frac{1}{1+\Omega_{f} / \sin ^{2} \theta} \exp \left(-\frac{\mu^{2}}{2 \sigma^{2}} \frac{\Omega_{f} / \sin ^{2} \theta}{1+\Omega_{f} / \sin ^{2} \theta}\right)\right]^{M N} d \theta,
\end{aligned}
$$

where

$$
\Omega_{f}=\frac{E_{s}}{4 N_{0}} 2 \sigma^{2} \frac{\beta}{M} \sum_{p=1}^{P}\left|x_{p}^{f}-\hat{x}_{p}^{f}\right|^{2} .
$$

To find the unconditional PEP, we still need to take an expectation of (13) with respect to $\mu$, whose distribution is given by (4). This expectation yields

$$
\begin{aligned}
P(\mathbf{X}, \hat{\mathbf{X}})=\frac{1}{\pi} \int_{\theta=0}^{\pi / 2} \prod_{f=1}^{F} & {\left[\frac{1}{1+\Omega_{f} / \sin ^{2} \theta} \frac{1}{\sqrt{2 \pi} \sigma_{\mu}}\right.} \\
& \times \int_{\mu=0}^{\infty} \frac{1}{\mu} \exp \left(-\frac{\mu^{2}}{2 \sigma^{2}} \frac{\Omega_{f} / \sin ^{2} \theta}{1+\Omega_{f} / \sin ^{2} \theta}\right) \\
& \left.\times \exp \left(-\frac{\left(\ln \mu-m_{\mu}\right)^{2}}{2 \sigma_{\mu}^{2}}\right) d \mu\right]^{M N} d \theta
\end{aligned}
$$

Introducing the variable change $u=\left(\ln \mu-m_{\mu}\right) / \sqrt{2 \sigma_{\mu}^{2}}$, can be rewritten as

$$
\begin{aligned}
& P(\mathbf{X}, \hat{\mathbf{X}}) \\
& =\frac{1}{\pi} \int_{\theta=0}^{\pi / 2} \prod_{f=1}^{F}\left[\frac{1}{1+\Omega_{f} / \sin ^{2} \theta} \frac{1}{\sqrt{\pi}}\right. \\
& \times \int_{u=-\infty}^{\infty} \exp \left(-u^{2}\right) \\
& \times \exp \left(-\frac{1}{2 \sigma^{2}} \frac{\Omega_{f} / \sin ^{2} \theta}{1+\Omega_{f} / \sin ^{2} \theta}\right. \\
& \left.\left.\times \exp \left(2 \sqrt{2} \sigma_{\mu} u+2 m_{\mu}\right)\right) d u\right]^{M N} d \theta \text {. }
\end{aligned}
$$

The inner integral has the form of $\int_{-\infty}^{\infty} \exp \left(-u^{2}\right) f(u) d u$, which can be expressed in terms of an infinite sum (see the appendix). This yields the final form of the exact PEP as

$$
\begin{aligned}
P(\mathbf{X}, \hat{\mathbf{X}})=\frac{1}{\pi} \int_{\theta=0}^{\pi / 2} \prod_{f=1}^{F}\{ & \frac{1}{1+\Omega_{f} / \sin ^{2} \theta} \exp \left(-\Delta_{f}(\theta)\right) \\
\times & {\left[1+\sum_{\substack{k=2 \\
k \text { even }}}^{\infty} \frac{(k-1) ! !}{k !}\left(2 \sigma_{\mu}\right)^{k}\right.} \\
& \left.\left.\times \sum_{d=1}^{k} g_{k, d}\left(\Delta_{f}(\theta)\right)^{d}\right]\right\}^{M N} d \theta,
\end{aligned}
$$


where

$$
\Delta_{f}(\theta)=\frac{1}{2 \sigma^{2}} \frac{\Omega_{f} / \sin ^{2} \theta}{2 \sigma^{2} 1+\Omega_{f} / \sin ^{2} \theta} \exp \left(2 m_{\mu}\right)
$$

and $(k-1) ! !=1.3 \cdots k$ [11, page xlv $]$. The coefficients $g_{k, d}$ in (17) can be computed by the recursive equation given in the appendix. It is worth noting that even considering only the first term in the infinite summation in (17) gives a very good approximation for practical values of shadowing. Setting $k=2$ and noting that $g_{2,1}=-1$ and $g_{2,2}=1$, we have

$$
\begin{aligned}
P(\mathbf{X}, \hat{\mathbf{X}}) \cong \frac{1}{\pi} \int_{\theta=0}^{\pi / 2} \prod_{f=1}^{F}\left\{\frac{1}{1+\Omega_{f} / \sin ^{2} \theta} \exp \left(-\Delta_{f}(\theta)\right)\right. \\
\left.\times\left[1-2 \sigma_{\mu}^{2} \Delta_{f}(\theta)+2 \sigma_{\mu}^{2}\left(\Delta_{f}(\theta)\right)^{2}\right]\right\}^{M N} .
\end{aligned}
$$

In our numerical results, taking more terms (i.e., $k>2$ ) did not result in a visible change in the plots.

It is also interesting to point out how (17) relates to the unshadowed case. Assuming there is no shadowing, $\mu$ is no longer a log-normal random variable, but just given as a constant equal to its mean $\mu=\exp \left(2 m_{\mu}\right)$. Furthermore, inserting $\sigma_{\mu}^{2}=0$ in (17) and using the relationships $\sigma^{2}=0.5 /(1+K)$ and $\mu=\sqrt{K /(1+K)}$ in terms of the well-known Rician parameter $K$, we obtain

\section{$P(\mathbf{X}, \hat{\mathbf{X}})$}

$$
\begin{aligned}
& =\frac{1}{\pi} \int_{\theta=0}^{\pi / 2} \prod_{f=1}^{F}\left[\frac{1+K}{1+K+\left(E_{s} / 4 N_{0}\right)\left(\beta / \sin ^{2} \theta\right) \sum_{p=1}^{P}\left|x_{p}^{f}-\hat{x}_{p}^{f}\right|^{2}}\right. \\
& \left.\times \exp \left(-\frac{K\left(E_{s} / 4 N_{0}\right)\left(\beta / \sin ^{2} \theta\right) \sum_{p=1}^{P}\left|x_{p}^{f}-\hat{x}_{p}^{f}\right|^{2}}{1+K+\left(E_{s} / 4 N_{0}\right)\left(\beta / \sin ^{2} \theta\right) \sum_{p=1}^{P}\left|x_{p}^{f}-\hat{x}_{p}^{f}\right|^{2}}\right)\right]^{M N} d \theta,
\end{aligned}
$$

which was previously presented in [5]. It is also interesting to note that simply by setting $\theta=\pi / 2$ in (17) and (20), the classical Chernoff bound would be obtained for shadowed and unshadowed Rician channels, respectively.

For sufficiently large signal-to-noise ratios (i.e., $E_{s} / N_{0} \gg$ 1 ), evaluating the integrand in (17) at $\theta=\pi / 2$, we obtain a Chernoff-type bound as

\section{$P(\mathbf{X}, \hat{\mathbf{X}})$}

$$
\leq\left(\frac{E_{s}}{4 N_{0}}\right)^{-|\Psi| N M} \prod_{f=1}^{|\Psi|}\left(\frac{\beta}{M} \sum_{P=1}^{P}\left|x_{P}^{f}-\hat{x}_{P}^{f}\right|^{2}\right)^{-N M}\left[q\left(\sigma, \sigma_{\mu}, m_{\mu}\right)\right]^{|\Psi| N M},
$$

where

$$
\begin{aligned}
& q\left(\sigma, \sigma_{\mu}, m_{\mu}\right) \\
& =\frac{1}{2 \sigma^{2}} \exp \left(-\frac{1}{2 \sigma^{2}} \exp \left(2 m_{\mu}\right)\right) \\
& \quad \times\left[1+\sum_{\substack{k=2 \\
k \text { :even }}}^{\infty} \frac{(k-1) ! !}{k !}\left(2 \sigma_{\mu}\right)^{k} \sum_{d=1}^{k} g_{k, d}\left(\frac{1}{2 \sigma^{2}} \exp \left(2 m_{\mu}\right)\right)^{d}\right] .
\end{aligned}
$$

Here, $\Psi$ is the set of inner frames (with a length of $L$ symbols) at nonzero Euclidean distance summations and $|\Psi|$ is the number of elements in this set. This can be compared to effective length (EL) in TCM schemes [12], which is defined as the smallest number of symbols at nonzero Euclidean distances. Contrary to the symbol-by-symbol count in the definition of EL, frame-by-frame count is considered here as a result of the multidimensional structure of STBC spanning an interval of $L$ symbols. It should also be noted that symbolby-symbol interleaving is considered for the single antenna case while an $L$-symbol interleaver is employed in our case. In (21), the slope of the performance curve, which yields the diversity order, is determined by $|\Psi| N M$ and it can be defined as generalized effective length (GEL) for multiple antenna systems in an analogy to the effective length for single antenna case.

The second term in (21) contributes to the coding gain, which corresponds to the horizontal shift in the performance curve. Recalling the definition of product distance (PD) for the single antenna case (which is given as the product of nonzero branch distances along the error event), we now define the generalized product distance (GPD)

$$
\prod_{f=1}^{|\Psi|}\left(\frac{\beta}{M} \sum_{p=1}^{P}\left|x_{p}^{f}-\hat{x}_{p}^{f}\right|^{2}\right)^{-N M}
$$

which involves the product of nonzero branch distance summations, where the summation is over $P$ terms based on the STBC used.

The third term in (21) is completely characterized by channel parameters. Since maximization of diversity order is the primary design criterion, the first step in "good" code design is the maximization of $|\Psi|$, since $M$ and $N$ are already fixed. Once diversity order is optimized, the third term becomes just a constant. This makes us conclude that the GEL and GPD are the appropriate performance criteria in the selection of trellis codes over shadowed Rician channels. This also shows that the trellis codes designed for optimum performance (based on classical effective code length and minimum product distance) over fading channels for the single transmit antenna case are not necessarily optimum for the multiple antenna case.

To derive the upper bound on bit error probability from the exact PEP, we follow the classical transfer function approach. The upper bound is given in terms of the transfer 


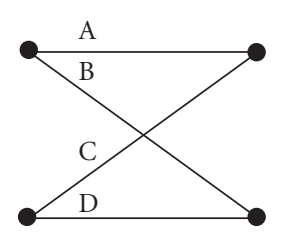

$$
A=\left[\begin{array}{l}
s_{0}, s_{0} \\
s_{0}, s_{4} \\
s_{2}, s_{2} \\
s_{2}, s_{6} \\
s_{4}, s_{0} \\
s_{4}, s_{4} \\
s_{6}, s_{2} \\
s_{6}, s_{6}
\end{array}\right] \quad B=\left[\begin{array}{l}
s_{0}, s_{2} \\
s_{0}, s_{6} \\
s_{2}, s_{0} \\
s_{2}, s_{4} \\
s_{4}, s_{2} \\
s_{4}, s_{6} \\
s_{6}, s_{0} \\
s_{6}, s_{4}
\end{array}\right] \quad C=\left[\begin{array}{l}
s_{1}, s_{3} \\
s_{1}, s_{7} \\
s_{3}, s_{1} \\
s_{3}, s_{5} \\
s_{5}, s_{3} \\
s_{5}, s_{7} \\
s_{7}, s_{1} \\
s_{7}, s_{5}
\end{array}\right] \quad D=\left[\begin{array}{l}
s_{1}, s_{1} \\
s_{1}, s_{5} \\
s_{3}, s_{3} \\
s_{3}, s_{7} \\
s_{5}, s_{5} \\
s_{5}, s_{1} \\
s_{7}, s_{7} \\
s_{7}, s_{3}
\end{array}\right]
$$

(a)

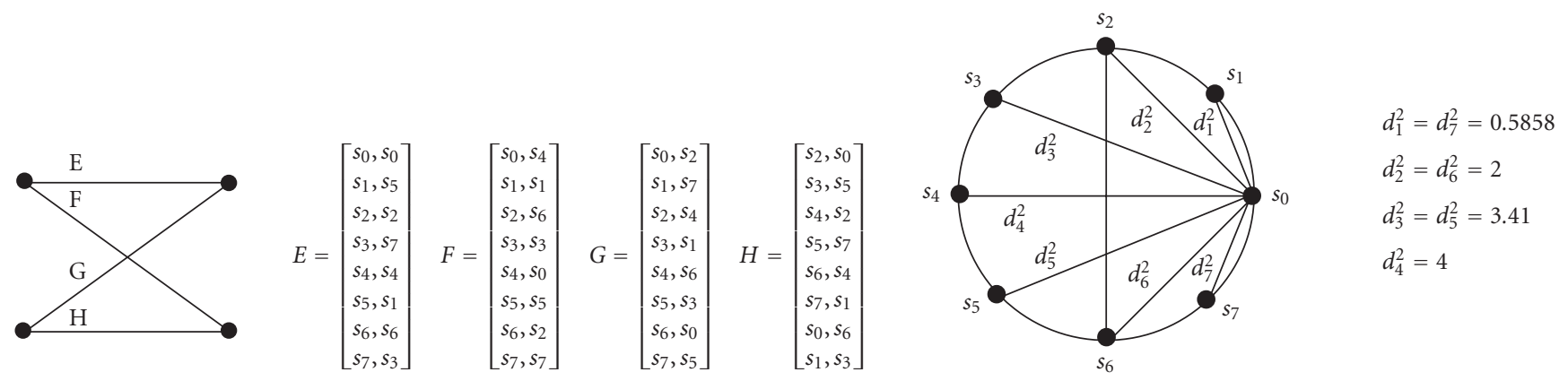

(b)

(c)

Figure 1: (a) Code A2, optimum for AWGN, (b) Code F2, optimum for Rayleigh fading channels with one transmit antenna, (c) 8-PSK signal constellation.

function of the code $T(D, I)$ by $[8,12]$

$$
P_{b} \leq\left.\frac{1}{\pi} \int_{0}^{\pi / 2} \frac{1}{n_{b}} \frac{\partial}{\partial I} T(D(\theta), I)\right|_{I=1} d \theta
$$

where $n_{b}$ is the number of input bits per transition and $T(D(\theta), I)$ is the modified transfer function of the code, where $D(\theta)$, is given in our case, by

$$
\begin{aligned}
D(\theta)= & \left(1+\frac{\Omega_{f}}{\sin ^{2} \theta}\right)^{-M N} \exp \left(-M N \Delta_{f}(\theta)\right) \\
& \times\left[1+\sum_{\substack{k=2 \\
k \text { :even }}}^{\infty} \frac{(k-1) ! !}{k !}\left(2 \sigma_{\mu}\right)^{k} \sum_{d=1}^{k} g_{k, d}\left(\Delta_{f}(\theta)\right)^{d}\right]^{M N}
\end{aligned}
$$

based on the derived PEP in (17).

\section{EXAMPLES}

In this section, we consider two different TCM schemes as outer codes whose trellis diagrams are illustrated in Figure 1. These are 2-state 8-PSK-MTCM codes with 2 symbols per branch, which are optimized for best performance over AWGN and Rayleigh fading channels, respectively [12]. For convenience, we summarize the important parameters of these codes from [12]. The free distance of the code A2 is $d_{\text {free }}^{2}=3.172$. Its minimum EL is determined by the error event path of $\left\{s_{0}, s_{4}\right\}$, which differs by one symbol from the correct path (the all-zeros path is assumed to be the correct path based on the uniform properties of the code) achieving
TABLE 1: Parameters for various degrees of shadowing.

\begin{tabular}{c|ccc}
\hline Parameter & Light & Average & Heavy \\
\hline$\sigma^{2}$ & 0.158 & 0.126 & 0.0631 \\
$m_{\mu}$ & 0.115 & -0.115 & -3.91 \\
$\sigma_{\mu}$ & 0.115 & 0.161 & 0.806 \\
\hline
\end{tabular}

$\mathrm{EL}=1$. The corresponding PD is $d_{4}^{2}=4$. On the other hand, the code $\mathrm{F} 2$ has a free distance of $d_{\text {free }}^{2}=2.343$ and it achieves $\mathrm{EL}=2$ with a product distance of $d_{1}^{2} \times d_{5}^{2}=2$, which is determined by the error event path of $\left\{s_{1}, s_{5}\right\}$. Since EL is the primary factor affecting performance (PD as a secondary factor) over fading channels, F2 is expected to have better performance than A2.

As an example of the shadowed Rician model, we consider the Canadian mobile satellite channel [6]. Table 1 shows the values of shadowing parameters for this channel, which are determined by empirical fit to measured data within Canada. In this table, the terms light, average, and heavy are used to represent an increasing effect of the shadowing.

The upper bounds for both codes with the single transmit antenna are illustrated in Figure 2. No STBC is considered in this case. As expected for the single transmit antenna case, F2 performs better than A2, where the performance is determined by the choices of EL and PD. This observation holds for all considered degrees of shadowing.

In Figure 3, upper bounds for the concatenated scheme are illustrated. Here we use the STBC designed for 2-TX antenna (i.e., Alamouti's code). Based on this code, we have $P=L=M=2$ and $\beta=1$. Our results demonstrate that 


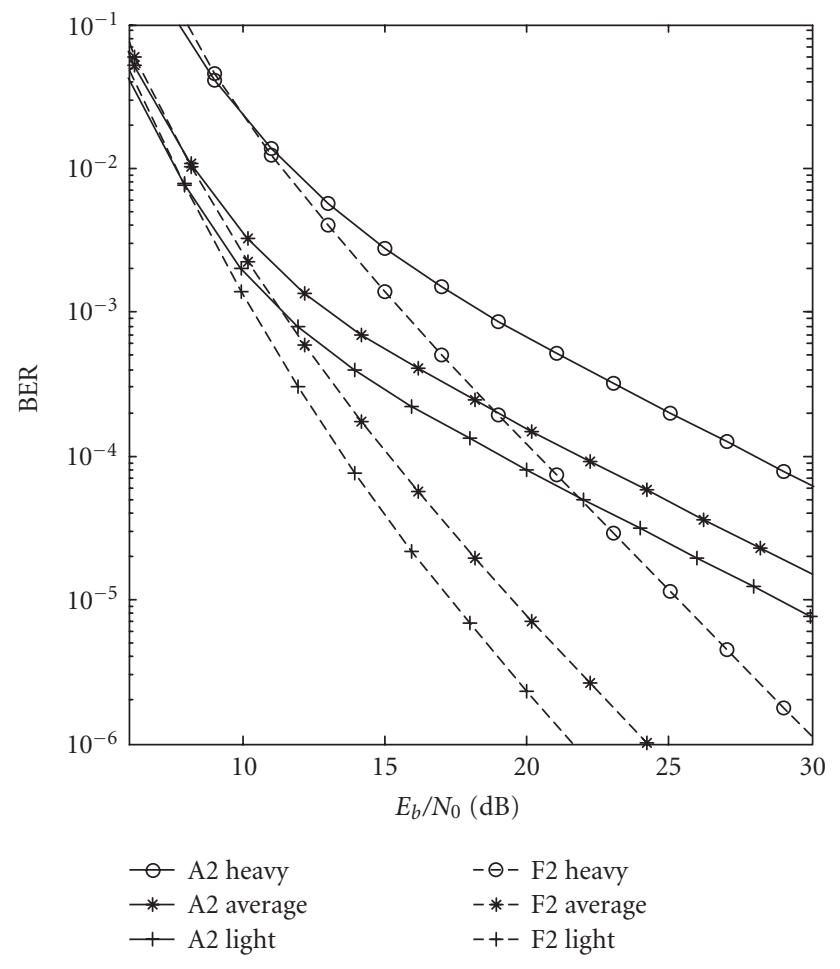

Figure 2: Upper bounds for codes A2 and F2 with single transmit antenna over shadowed Rician channels (1-TX and 1-RX antenna).

the concatenated schemes using A2 and F2 as outer trellis codes achieve roughly the same performance. This is a result of the fact that the dominant factors for the single antenna case no longer determine performance. In the 2-TX antenna case, both schemes achieve GEL equal to 2 and GPD equal to 4 , that is, $\left[\left(d_{1}^{2}+d_{5}^{2}\right) / 2\right]^{2}=4$ for F2 and $\left[\left(d_{0}^{2}+d_{4}^{2}\right) / 2\right]^{2}=4$ for A2, based on (23). Since both of them have equal GEL and GPD, their performances turn out to be almost identical. This observation holds to be true independent of considered degrees of shadowing.

Comparison between the one- and two-transmitantenna cases also reveals interesting points on the performance. In both figures, code F2 gives a diversity order of 2 (i.e., slope of the curve), regardless of antenna numbers. Only an additional coding gain (i.e., horizontal shift in the curve) is observed with the use of two antennas. However, this result is somewhat a coincidence because of the particular choice of the parameters characterizing this specific example. For the single transmit antenna case, the code $\mathrm{F} 2$ has $\mathrm{EL}=2$ and the performance curve varies with $\left(E_{b} / N_{0}\right)^{-2}$. On the other hand, for the 2-TX antenna case we have $|\Psi|=1$, since an $L=2$ symbol interleaver is used. However, the overall diversity is determined by GEL (i.e., $|\Psi| N M=1 \cdot 1 \cdot 2=2$ ), resulting again in the same slope as in the single transmit antenna case.

To examine the tightness of upper bounds, we also evaluate the performance of codes A2 and F2 through computer simulation, assuming 2-TX antennas. Simulation results for the code F2 are illustrated in Figure 4 with the corresponding

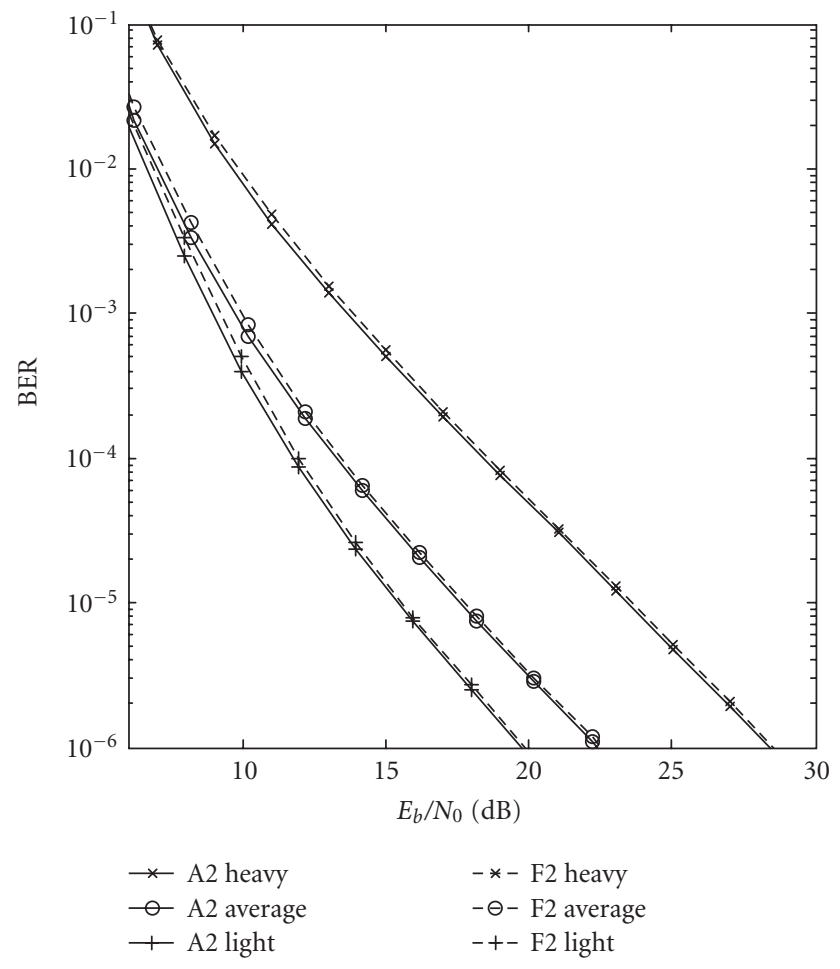

FIGURE 3: Upper bounds for concatenated MTCM-STBC schemes with codes A2 and F2 as outer codes over shadowed Rician channels (2-TX and 1-RX antenna).

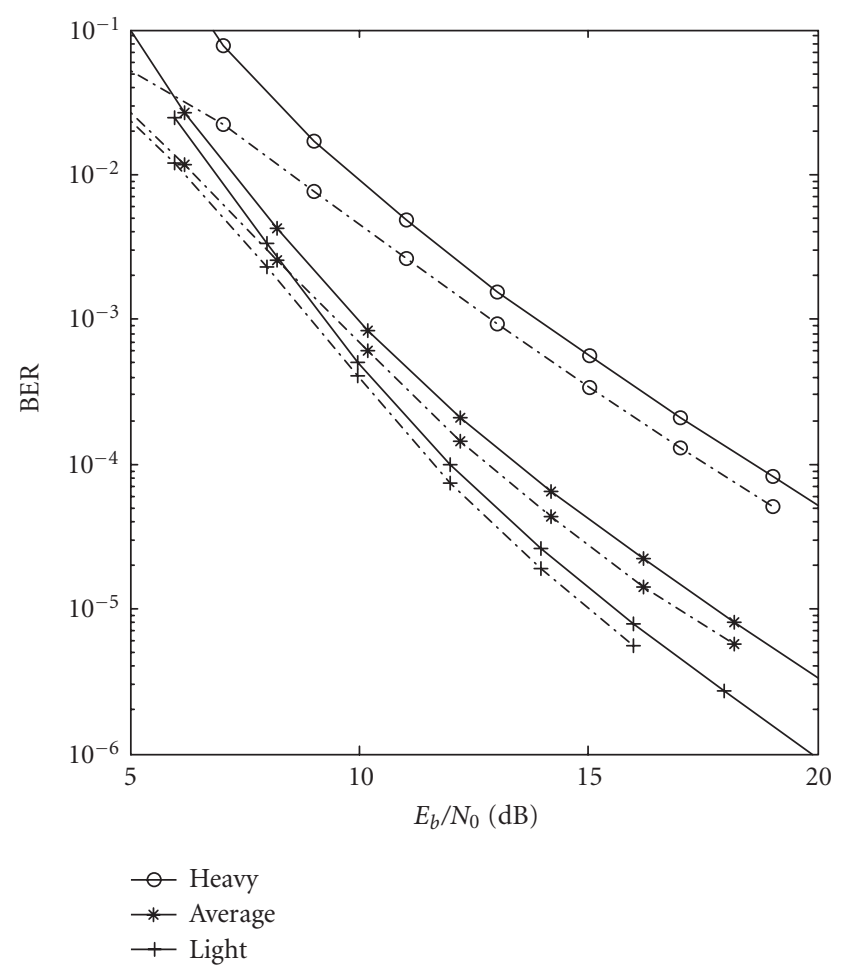

FIgURE 4: Upper bounds versus simulation results for code F2 (solid: upper bounds, dashed: simulation). 
upper bounds (plotted as solid lines) computed by (24) and (25). The upper bounds are in very good agreement with simulation results, demonstrating the tightness of the new upper bounds based on the exact PEP. As expected (based on our previous discussion on upper bound expressions), code A2 yields nearly identical simulation results to those of code F2, which we do not include here for brevity.

\section{CONCLUSION}

We analyzed the performance of trellis-coded STBC schemes over shadowed Rician fading channels. Our analysis is based on the derivation of an exact PEP through the moment generating function approach. The derived expression provides insight into the selection criteria for trellis codes which should be used in conjunction with STBC over fading channels. Our results also show that the trellis codes designed for optimum performance over Rician channels with single transmit antenna are not necessarily optimum for the multiple transmit antenna case. Using transfer function techniques based on the new PEP, we present upper bounds on the bit error probability for the concatenated scheme. We also provide simulation results, which seem to be in good agreement with the derived upper bounds.

\section{APPENDIX}

This appendix evaluates the inner integral in (16) in terms of an infinite sum. Defining

$$
a=\frac{1}{2 \sigma^{2}} \frac{\Omega_{f} / \sin ^{2} \theta}{1+\Omega_{f} / \sin ^{2} \theta}, \quad b=2 \sqrt{2} \sigma_{\mu}, \quad c=2 m_{\mu},
$$

we can write the inner integral in (16) as

$$
\int_{-\infty}^{\infty} \exp \left(-u^{2}\right) f(u) d u
$$

with $f(u)=\exp (-a \exp (b u+c))$. Expanding $f(u)$ in Taylor series, we obtain

$$
\int_{-\infty}^{\infty} \exp \left(-u^{2}\right) f(u) d u=\sum_{k=0}^{\infty} \frac{f^{k}(0)}{k !} \int_{-\infty}^{\infty} u^{k} \exp \left(-u^{2}\right) d u
$$

where $f^{k}(0)$ are the Taylor series coefficients and, in our case, they can be determined as

$$
f^{k}(0)=\exp (-a \exp (c)) b^{k} \sum_{d=1}^{k} g_{k, d}(a \exp (c))^{d}
$$

where $g_{k, d}$ can be computed by the recursive equation

$$
\begin{gathered}
g_{k, d}=d g_{k-1, d}-g_{k-1, d-1} \quad \text { with } g_{k, 1}=-1 \text { for } k=1,2, \ldots, \\
g_{k, d}=0 \quad \text { for } d>k .
\end{gathered}
$$

Using the integral form given by [11, page 382, equation 3.462.1], it can easily be shown that the integral in (A.3) is zero for the odd values of $k$. For even values of $k$, we can use the result [11, page 382, equation 3.461.4] and express (A.3) as

$$
\int_{-\infty}^{\infty} \exp \left(-u^{2}\right) f(u) d u=\sqrt{\pi} \sum_{k=0}^{\infty} \frac{f^{k}(0)}{k !} \frac{(k-1) ! !}{2^{k / 2}} .
$$

Replacing (A.2) by (A.6) with $a, b$, and $c$ values given as in (A.1), one can obtain the final form for the inner integral of (16) leading to (17).

\section{ACKNOWLEDGMENT}

This paper was presented in part at IEEE Vehicular Technology Conference (VTC-Fall '02), Vancouver, Canada, October 2002.

\section{REFERENCES}

[1] V. Tarokh, N. Seshadri, and A. R. Calderbank, "Space-time codes for high data rate wireless communication: performance criterion and code construction," IEEE Transactions on Information Theory, vol. 44, no. 2, pp. 744-765, 1998.

[2] V. Tarokh, H. Jafarkhani, and A. R. Calderbank, "Space-time block codes from orthogonal designs," IEEE Transactions on Information Theory, vol. 45, no. 5, pp. 1456-1467, 1999.

[3] S. M. Alamouti, "A simple transmit diversity technique for wireless communications," IEEE Journal on Selected Areas in Communications, vol. 16, no. 8, pp. 1451-1458, 1998.

[4] S. M. Alamouti, V. Tarokh, and P. Poon, "Trellis-coded modulation and transmit diversity: design criteria and performance evaluation," in Proc. IEEE 1998 International Conference on Universal Personal Communications, vol. 1, pp. 703-707, Florence, Italy, October 1998.

[5] M. Uysal and C. N. Georghiades, "Analysis of concatenated trellis coded STBC schemes over Rician fading channels," in Proc. 39th Annual Allerton Conference on Communication, Control and Computing, Monticello, Ill, USA, October 2001.

[6] C. Loo, "A statistical model for a land mobile satellite link," IEEE Trans. Vehicular Technology, vol. 34, no. 3, pp. 122-127, 1985.

[7] M. K. Simon and M.-S. Alouini, "A unified approach to the performance analysis of digital communication over generalized fading channels," Proceedings of the IEEE, vol. 86, no. 9, pp. 1860-1877, 1998.

[8] M. K. Simon and M.-S. Alouini, Digital Communication over Fading Channels: A Unified Approach to Performance Analysis, John Wiley \& Sons, New York, NY, USA, 2000.

[9] G. L. Turin, "The characteristic function of Hermitian quadratic forms in complex normal variables," Biometrika, vol. 47, no. 1/2, pp. 199-201, 1960.

[10] A. M. Mathai and S. B. Provost, Quadratic Forms in Random Variables: Theory and Applications, Marcel Dekker, New York, NY, USA, 1992.

[11] I. S. Gradshteyn and I. M. Rzyhik, Table of Integrals, Series and Products, Academic Press, San Diego, Calif, USA, 5th edition, 1994.

[12] E. Biglieri, D. Divsalar, P. J. McLane, and M. K. Simon, Introduction to Trellis-Coded Modulation with Applications, Macmillan Publishing, New York, NY, USA, 1991. 
M. Uysal was born in Istanbul, Turkey, in 1973. He received the B.S. and the M.S. degrees in electronics and communication engineering from Istanbul Technical University, Istanbul, Turkey, in 1995 and 1998, respectively, and the Ph.D. degree in electrical engineering from Texas A\&M University, Texas, in 2001. From 1995 to 1998, he worked as a Research and Teaching Assistant in the Communication Theory Group

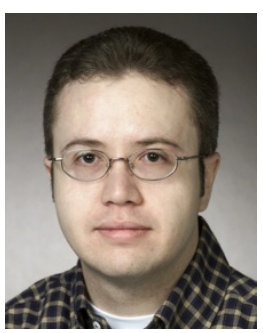
at Istanbul Technical University. From 1998 to 2002, he was affiliated to the Wireless Communication Laboratory, Texas A\&M University. During the fall of 2000, he worked as a Research Intern at AT\&T Labs-Research, New Jersey. In April 2002, he joined the Department of Electrical and Computer Engineering, University of Waterloo, Canada, as an Assistant Professor. His research interests lie in communications theory with special emphasis on wireless applications. Specific areas include space-time coding, diversity techniques, coding for fading channels, and performance analysis over fading channels. Dr. Uysal currently serves as an Editor for IEEE Transactions on Wireless Communications and as the Guest Coeditor for Special Issue on "MIMO Communications" of Wiley Journal on Wireless Communications and Mobile Computing.

C. N. Georghiades received his doctorate in electrical engineering from Washington University in May 1985. Since September 1985 he has been with the Electrical Engineering Department at Texas A\&M University where he is a Professor and holder of the Delbert A. Whitaker Endowed Chair. His general interests are in the application of information, communication, and estimation theories to the study of communication sys-

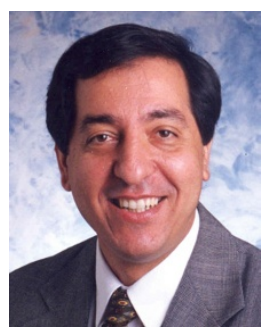

tems, with particular interest in wireless and optical systems. Dr. Georghiades has served over the years in several editorial positions with the IEEE Information Theory and Communication Societies and has been involved in organizing a number of conferences. He currently serves as Chair of the Fellow Evaluation Committee of the IEEE Information Theory Society and in the Awards Committee of the IEEE Communications Society. He also serves as General Cochair for the IEEE Information Theory Workshop in San Antonio, Texas, in October 2004. Dr. Georghiades was the recipient of the 1995 Texas A\&M University College of Engineering Halliburton Professorship and the 2002 E.D. Brockett Professorship. From 1997 to 2002 he held the J. W. Runyon Jr. Endowed Professorship and in 2002 he became the inaugural recipient of the Delbert A. Whitaker Endowed Chair. 